\title{
Caracterização granulométrica e mineralógica dos sedimentos como suporte para análise de contaminação ambiental em nascentes do rio Subaé, Feira de Santana (BA)
}

\begin{abstract}
Maria Alexandra Santivanez Cruz Leila Thaise Santana de Oliveira Santos Lilian Graicy Lima Morais Lima Taise Bomfim de Jesus*

Programa de Pós-Graduação em Modelagem em Ciências da Terra e do Ambiente, Universidade Estadual de Feira de Santana (UEFS) Feira de Santana (BA), Brasil.
\end{abstract}

*Autor correspondente: taisebj@hotmail.com

\section{Resumo}

No presente artigo são apresentadas as composições mineralógica e granulométrica de sedimentos coletados nas nascentes da bacia do rio Subaé, Feira de Santana, Bahia, como ferramenta de suporte para futuros trabalhos de avaliação geoquímica dos sedimentos das nascentes do rio Subaé. Análises mineralógicas e granulométricas, além de indicarem a hidrodinâmica do sistema e das condições ambientais de sedimentação, também são fatores importantes no controle da distribuição natural e/ou antrópica dos elementos químicos nos sedimentos. Neste trabalho, a análise granulométrica das amostras obedeceu ao protocolo referente às normas brasileiras de análise NBR 7181 e a determinação da distribuição granulométrica dos sedimentos finos foi feita com ensaio de sedimentação. A determinação das fases minerais foi feita utilizando um espectrorradiômetro portátil modelo ASD-FieldSpec Full Resolution 3, em laboratório. Foi observada grande variação no teor de matéria orgânica $(2,0$ a $28,4 \%$ ) obtido nos diferentes pontos de coleta; esses resultados implicam na qualidade dos sedimentos, uma vez que grande porcentagem dos elementos metálicos (nutrientes e/ou tóxicos) é adsorvida pela matéria orgânica. Os sedimentos superficiais das nascentes do rio Subaé apresentaram indícios de alta capacidade adsortiva de metais por serem de textura argilosa, porém apresentaram predomínio de minerais do tipo caulinita e esmectita, conhecidas como argilas de baixa capacidade de troca catiônica. Tais características são importantes para o tamponamento do solo aos metais pesados e para diminuir o risco de contaminação do nível hidrostático e de cursos d'água.

Palavras-chave: mineralogia, granulometria, sedimentos, contaminação, nascentes.

\section{Abstract}

In this article it is discussed the mineralogical composition and grain size of sediment collected in the headwaters of the Subae river basin, in Feira de Santana, Babia as a support tool for future geochemistry evaluation of sediments from the nascent of the river Subaé. Granulometric and mineralogical analyses, more than indicating the hydrodynamics of the system and environmental conditions of sedimentation, are also important factors in controlling the natural and/or anthropogenic chemical elements in sediments. In this research, the particle size analysis of the samples followed the protocol concerning the standards in Brazil (NBR 7181) in which the analysis and determination of particle size distribution of fine sediments is done with sedimentation tests. The determination of mineral layers was performed using a portable spectroradio- 
meter model FieldSpec Full Resolution ASD-3, in the laboratory. We observed large variations in organic matter obtained in the different collection points $(2.0$ to $28.4 \%$ ). These results show that a large percentage of the metallic elements (nutrients and/or toxic) was adsorbed by organic matter influencing into the sediment quality. The surface sediments of the nascents of the Subae River showed evidence of high adsorptive capacity for metals because of their clayey texture, in which kaolinite and smectite predominate over larger grain-size particles. These are known as clays with low cation-exchange capacity. These characteristics are important to buffer the soil against heavy metals and to decrease the risk of contamination at a hydrostatic level and for water streams. Keywords: mineralogical; granulometric; sediments; contamination; headwaters.

\section{INTRODUÇÃO}

O município de Feira de Santana, Bahia, está localizado na zona de planície entre o Recôncavo baiano e os tabuleiros semiáridos do nordeste baiano. Possui uma área de $1.363 \mathrm{~km}^{2}$, sendo reconhecido como o portal do sertão por estar situado no início do agreste baiano. Sua principal bacia hidrográfica encontra-se localizada no Recôncavo Norte, em uma área de $655 \mathrm{~km}^{2}$. O principal curso d'água dessa bacia, o rio Subaé, tem suas nascentes localizadas no perímetro urbano de Feira de Santana e abrange uma área de $1.451,629$ ha com altitude média de $220 \mathrm{~m}$. A sua localização está definida pelas coordenadas $12^{\circ} 25^{\prime}$ e $12^{\circ} 28^{\prime}$ de latitude sul e 38 $90^{\prime}$ e $38^{\circ} 96^{\prime}$ de longitude oeste (Datum WGS/84).

De maneira geral, é possível definir que as nascentes do rio Subaé são do tipo aquífero confinado, com aspecto de lagoa, já que forma parte do manancial subterrâneo que aflora na superfície e se encontra entre duas camadas impermeáveis de material sólido (Franca-Rocha et al. 1998). Com exceção da nascente da Rua Pedro Suzart, a qual foi identificada por meio de sensoriamento remoto através de imagens de satélite LANDSAT 5 e através da observação sistêmica em campo, se constatou que é uma nascente sem acúmulo de água, perene, com um fluxo permanente que está atualmente recebendo as descargas domésticas da comunidade do Vietnã, Feira de Santana.

As interferências humanas nas nascentes da bacia do rio Subaé principiaram em 1970 com a instalação do Complexo Industrial do Subaé (CIS), inicialmente com 37 empresas, e hoje com pouco mais de 350 indústrias em operação. Tal desenvolvimento industrial exigiu outras alterações na paisagem da cidade como construções de estradas para transporte de produtos e/ou subprodutos, avanço do ramo imobiliário, entre outros. Todas essas atividades contribuem com as alterações na qualidade dos corpos hídricos da região. Apesar do desenvolvimento industrial, conservam-se ainda hoje pequenas áreas agrícolas nas áreas de nascentes (lagoas) do rio Subaé.

O substrato geológico da região de Feira de Santana é caracterizado por apresentar dois grandes conjuntos litológicos principais: o embasamento cristalino de idade pré-cambriana, variando entre 2,0-3,0 Ga e as coberturas sedimentares tércio-quaternárias (Barbosa \& Dominguez 1996). As rochas que compõem o embasamento cristalino são representadas por unidades litoestratigráficas do Bloco Jequié e Cinturão Móvel
Salvador-Curaçá, composto predominantemente por granitóides, gnáisses e migmatitos metamorfisados na fácies granulitos. O Grupo Barreiras, representado por arenitos finos a grossos com níveis argilosos e conglomeráticos, preenche paleovales (canais de antigos vales) e depressões, servindo como um divisor de águas das bacias do Jacuípe e Subaé. O material é bastante poroso e permeável sendo responsável pelo acúmulo e transmissão de águas subterrâneas, originando fontes e lagoas que geram as três micro bacias hidrográficas no município (Santos 2004).

Segundo Alloway \& Ayres (1997) sedimentos de fundo desempenham papel muito importante na avaliação da poluição de mananciais. Eles refletem a qualidade atual e/ou histórica, caso se avalie amostras em profundidade, do sistema aquático e podem ser usados para detectar a presença de contaminantes, principalmente os que não permanecem solúveis após o seu lançamento em águas superficiais. Além disso, os sedimentos podem agir como possíveis fontes de poluição, como por exemplo, de metais pesados, os quais podem não ser permanentemente fixados por eles, podendo ser novamente disponibilizados para a coluna d'água, através de variações nas condições ambientais tais como mudanças de $\mathrm{pH}$, de potencial redox, ou pela presença de quelantes orgânicos (Lemes et al. 2003).

Os substratos mais importantes na retenção de metais são aqueles que ocorrem naturalmente em grande quantidade, que possuem grandes áreas superficiais e elevada capacidade de troca catiônica. Nos sedimentos, esses substratos ativos são termodinamicamente instáveis, de natureza amorfa ou criptocristalina (Jones \& Bowser 1978). Os substratos mais comuns nos sedimentos de rio são óxidos de ferro e manganês, matéria orgânica e argilominerais (Horwitz 1991), sendo encontrados nos solos substratos similares. Os argilominerais são minerais secundários, formados pelo desgaste das rochas primárias, consistindo, geralmente, de óxido de alumínio hidratado e óxido de silício. Fisicamente, os argilominerais apresentam granulação muito fina e estrutura lamelar. O termo argila significa fração granulométrica e é aplicado a todas as partículas com diâmetro inferior a 0,004 mm, segundo Wentworth 1922 apud Suguio 1982, ou inferior a 0,002 mm, segundo Folk \& Ward (1957).

Os minerais de argila atuam como importantes coletores e concentradores de metais (Fostner \& Wittman 1981). 
As argilas são tipicamente materiais muito finos, com grandes áreas superficiais e capacidade de troca catiônica entre moderada a alta, isso, em função da sua carga superficial negativa. A elevada capacidade de troca catiônica e carga superficial são devidas a substituições isomorfas de íons na rede cristalina e ao rompimento de ligações em suas estruturas químicas (Gibbs 1977, Fisher et al. 1982, Salomons \& Forstner 1984). Os três principais grupos de argilominerais são: caolinita $\left(\mathrm{Al}_{2}(\mathrm{OH})_{4} \mathrm{Si}_{2} \mathrm{O}_{5}\right)$, ilita e montmorilonita $\left(\mathrm{Al}_{2}(\mathrm{OH})_{2} \mathrm{Si}_{4} \mathrm{O}_{10}\right)$ (Manahan 2009). A capacidade de sorver metais varia com o tipo da argila, como apresentado a seguir:

Montmorilonita $>$ Vermiculita $>$ Ilita $=$ Clorita $>$ Caulinita

O presente artigo tem por objetivo principal determinar a composição granulométrica e mineralógica, através de técnica espectrorradiométrica, de sedimentos nas nascentes da Bacia do rio Subaé, como ferramenta de suporte para futuros trabalhos de avaliação geoquímica dos sedimentos das nascentes do rio Subaé, uma vez que as regiões de nascentes desse rio estão em áreas consideradas periféricas e formam uma região de lagoas, mais suscetíveis e/ou sensíveis a contaminações químicas. É no entorno dessas lagoas que está alocado o Centro Industrial do Subaé (CIS), criado em 1969, que tem hoje uma produção diversificada, com 349 indústrias catalogadas em maio de 2012. Essas indústrias são especializadas na produção de bens de consumo final, como indústrias químicas, alimentícias, de galvanoplastia, dentre outras, além de pequenas e médias indústrias localizadas em diferentes pontos da cidade. A instalação desse centro industrial no município de Feira de Santana vem transformando consideravelmente o desenvolvimento econômico local, que está em constante crescimento.

\section{MATERIAIS E MÉTODOS}

\section{1. Área de Estudo}

A bacia do rio Subaé está localizada na região denominada de Recôncavo Norte no Estado da Bahia, inserida na região Hidrográfica Nacional Atlântico Leste, porém a área deste estudo limita-se ao alto curso da bacia que está inserido dentro do município de Feira de Santana (Figura 1), situado em uma zona entre o Agreste e o Semiárido (zona de planície entre o Recôncavo e os tabuleiros semiáridos do nordeste baiano). Tem uma altitude de 234 metros, com uma área de $1.338,1 \mathrm{~km}^{2}$. O clima é Seco a subúmido. A temperatura média anual do município de Feira de Santana é de $25^{\circ} \mathrm{C}$ e a pluviometria média anual é de $781 \mathrm{~mm}$, sendo os períodos chuvosos com predominância de concentração no quadrimestre de abril a julho com pico em junho (Brandão e Santos 2010).

Os pontos de amostragem escolhidos no presente estudo estão localizados nas áreas de nascentes da bacia hidrográfica do rio Subaé e estão apresentadas na Figura 1, nomeadas como: lagoa Salgada, representada na figura por LSA; lagoa Subaé do lado norte da BR-324, representada na figura por LSU A, e lagoa Subaé do lado sul da BR-324, representada na figura por LSU B; assim como o ponto referenciado pela base cartográfica como nascente, localizada no Bairro Brasília, na Rua Pedro Suzart, representada na figura por NPS.

Foram coletadas 14 amostras de sedimentos superficiais (primeiros $15 \mathrm{~cm}$ ) utilizando-se a draga do tipo Van Veen (em aço inox). As amostras foram acondicionadas em sacos plásticos e levadas ao Laboratório de Geoquímica da Universidade Estadual de Feira de Santana (UEFS), onde se

Figura 1

Localização dos pontos de coleta das amostras de sedimentos nas nascentes do rio Subaé, Feira de Santana, Bahia. As coordenadas estão em projeção UTM, datum WGS 84, zona 24S.

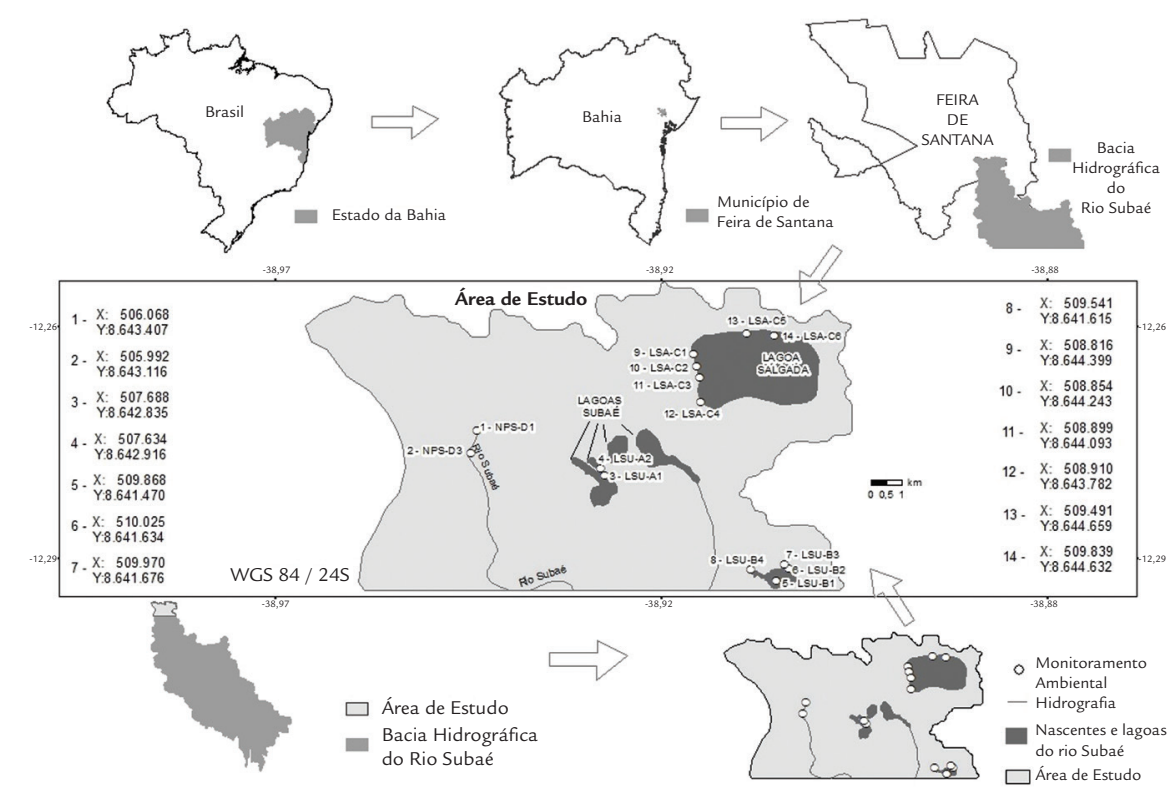


procederam às análises. As amostras foram secas em estufa a $60^{\circ} \mathrm{C}$. Em seguida, procedeu-se à maceração dos grãos (almofariz e pistilo com ponta de borracha) para o posterior quarteamento.

A análise granulométrica foi realizada seguindo-se a NBR 7181 da ABNT (1982). Para se determinar a distribuição granulométrica da fração dos sedimentos finos (partículas com diâmetros inferiores a $0,075 \mathrm{~mm}$ ), realizou-se o ensaio de sedimentação, através da velocidade de queda das partículas das amostras em um meio líquido, sendo que para isso tomou-se como base a lei de Stokes, que correlaciona a velocidade de queda das partículas esféricas com o seu diâmetro. Para a apresentação dos resultados, utilizou-se como recurso o SYSGRAM ${ }^{\circledast}$ para se confeccionar o Diagrama de Shepard e dendogramas.

A determinação da matéria orgânica $(\mathrm{MO})$ foi realizada na fração final $(0,075 \mathrm{~mm})$ feita pelo método gravimétrico segundo Wetsel \& Likens (1991). As amostras foram resfriadas em dessecador por uma hora, de modo que a interferência da umidade do ar fosse minimizada. Foram utilizados cadinhos de porcelana previamente lavados, secos, identificados e tarados para a pesagem de $0,1 \mathrm{~g}$ de sedimento seco, em balança analítica Scaltec SBC 31. A ignição em mufla foi à temperatura de $550^{\circ} \mathrm{C}$ por um período de quatro horas. $\mathrm{O}$ resfriamento foi feito no dessecador por duas horas. Em seguida os cadinhos foram pesados novamente. $\mathrm{O}$ teor de matéria orgânica foi determinado através da diferença entre o peso seco de sedimento e o peso após a ignição, considerando o peso seco (\% PS), conforme a equação abaixo:

$\% \mathrm{MO}=($ Peso sego g - Peso após a ignição g) x100 Peso sego g

Para a caracterização mineralógica foi utilizado o espectrorradiômetro portátil modelo ASD-FieldSpec Full

\section{RESULTADOS E DISCUSSÃO}

\subsection{Análise Granulométrica}

Os diagramas triangulares são de grande aplicação na sedimentologia, pois, além de representarem atributos granulométricos também representam características mineralógicas. Um gráfico triangular poderá indicar as contribuições relativas de cada uma das rochas matrizes dos sedimentos (Souza 2011). O gráfico utilizado neste trabalho foi o diagrama triangular de classificação de sedimentos clásticos, de acordo com a granulometria de Shepard (1954).

A classificação dos sedimentos de grãos finos, segundo o Diagrama de Shepard e dendograma das amostras arenosas, seguindo a norma NBR- 6502/95 são mostrados nas Figuras 2 e 3.

Segundo Camargo (2006), na representação gráfica da composição granulométrica dos sedimentos no SysGram, muitas amostras plotadas simultaneamente podem gerar gráficos confusos. Algumas amostras da lagoa salgada (LSA) aparecem sobrepostas por serem de composições granulométricas mui-
Resolution 3, que gerou os espectros de reflectância em laboratório. Para as medidas de refletância utilizou-se como acessório a sonda de contato High Intensity Contact Probe, que possui iluminação interna, não sendo necessário utilizar iluminação adicional. A preparação da amostra para essas análises consistiu em peneiramento em peneira de $2 \mathrm{~mm}$ sendo, em seguida, acondicionadas em placas de petri.

O procedimento para análise espectral das curvas de reflectância foi realizado de acordo com a metodologia estabelecida por Pontual et al. (1997) sendo constituída das seguintes etapas: a) aplicação da remoção de ruídos, visando se acentuar os picos de absorção; b) observação da região espectral do infravermelho de ondas curtas (SWIR) compreendida entre $2.050-2.450 \mathrm{~nm}$, visto que é nessa região que ocorrem as principais assinaturas espectrais dos minerais; c) identificação da feição de absorção mais profunda nessa região espectral, observando a posição da feição de absorção, tipo de feição (simples, dupla, múltipla), simetria, intensidade, forma e declividade e d) utilização de uma biblioteca espectral de referência para comparação do espectro da amostra com espectros conhecidos.

A análise espectral foi realizada tendo como suporte o software ENVI $4.5^{\circledast}$ para visualização das curvas e comparação com espectros da biblioteca de referência. Também foi utilizado o software SpecMin ${ }^{\circledR}$ (versão demo) para identificação das principais picos de absorção. A biblioteca de referência utilizada foi a da United States Geological Survey (USGS), que é constituída por espectros de minerais puros. Os principais picos de absorção encontrados, na região espectral do SWIR, foram comparados com as bandas de absorção encontradas por Hunt (1977), Hunt \& Ashley (1979), Grove et al. (1992), Pontual et al. (1997) e Clark (1999). Não foram realizadas outras técnicas de detecção mineralógica para comparação dos resultados obtidos na espectrorradiometria. to semelhantes entre elas. Os resultados nos diagramas são mostrados através de triângulos de diversos tamanhos, o que representa a quantidade de grânulos na amostra, sendo essa a quarta variável no triângulo. Quando a quantidade é muito pequena $(<3 \%)$ o triângulo também é pequeno e não aparece, sendo substituído por um círculo, como é o caso das amostras LSU-B4, LSU-B1 e LSA-C6, como se observa na Figura 2.

A partir desta representação gráfica (Figura 2) é possível observar a dominância das classes argila arenosa, característica da área da Lagoa Salgada e areia argilosa característica da área da Lagoa Subaé. A Lagoa Subaé norte (LSU), apresentou amostras com significativa variação na textura, desde argila-síltico-arenosa, no ponto LSU-B1, até areia no ponto LSU-B3. Essa variação na textura em uma pequena área com pontos de coleta próximos entre si pode ser explicada pelos impactos ambientais na área de estudo. Esses impactos estão relacionados com a retirada da vegetação original, a retirada de 
sedimentos para uso em olarias nas construções civis e aterramento das lagoas para construção residencial e industrial, suprimindo parte da área da lagoa. Tais impactos apresentam-se intensos e, de certo modo, irreversíveis em decorrência da forma como o homem interagiu no meio-ambiente, evidenciando interferências e mudanças na geomorfologia das lagoas. A utilização desse espaço durante as últimas décadas (1980-2010) provocou a descaracterização desse ecossistema lacustre. Assim, nota-se a diminuição da área, fazendo com que em época de chuva ás águas ultrapassem o limite imposto pela urbanização, gerando problemas ambientais, além da alteração da qualidade físico-química e biológica da água da lagoa.

Em sedimentos coletados nos pontos NPS-D1, NPS-D3 e LSU-A1, a fração areia foi preponderante, com frações de grânulos menores a $0,075 \mathrm{~mm}$, sendo assim, elas foram excluídas do diagrama triangular de Shepard. Para as frações granulométricas dos sedimentos coletados nesses pontos, adotou-se a confecção de histogramas em porcentagem, conforme mostra a Figura 3.

Segundo a classificação textural das amostras NPS-D1 e NPS-D3, essas são predominantemente areia média, sendo que no caso da Lagoa Subaé (Amostra LSU-A1), areia média com muitos grânulos. A distribuição granulométrica, nesses ambientes, reflete o uso e a ocupação desses locais, principalmente na época deste estudo, pois a empresa de esgotamento sanitário do estado estava realizando obras nesses locais e usava constantemente esses ambientes para despejar o material usado para essas obras.
Figura 2

Diagrama de Shepard contendo os resultados das análises granulométricas das amostras de sedimento das nascentes do rio Subaé, Feira de Santana (BA).
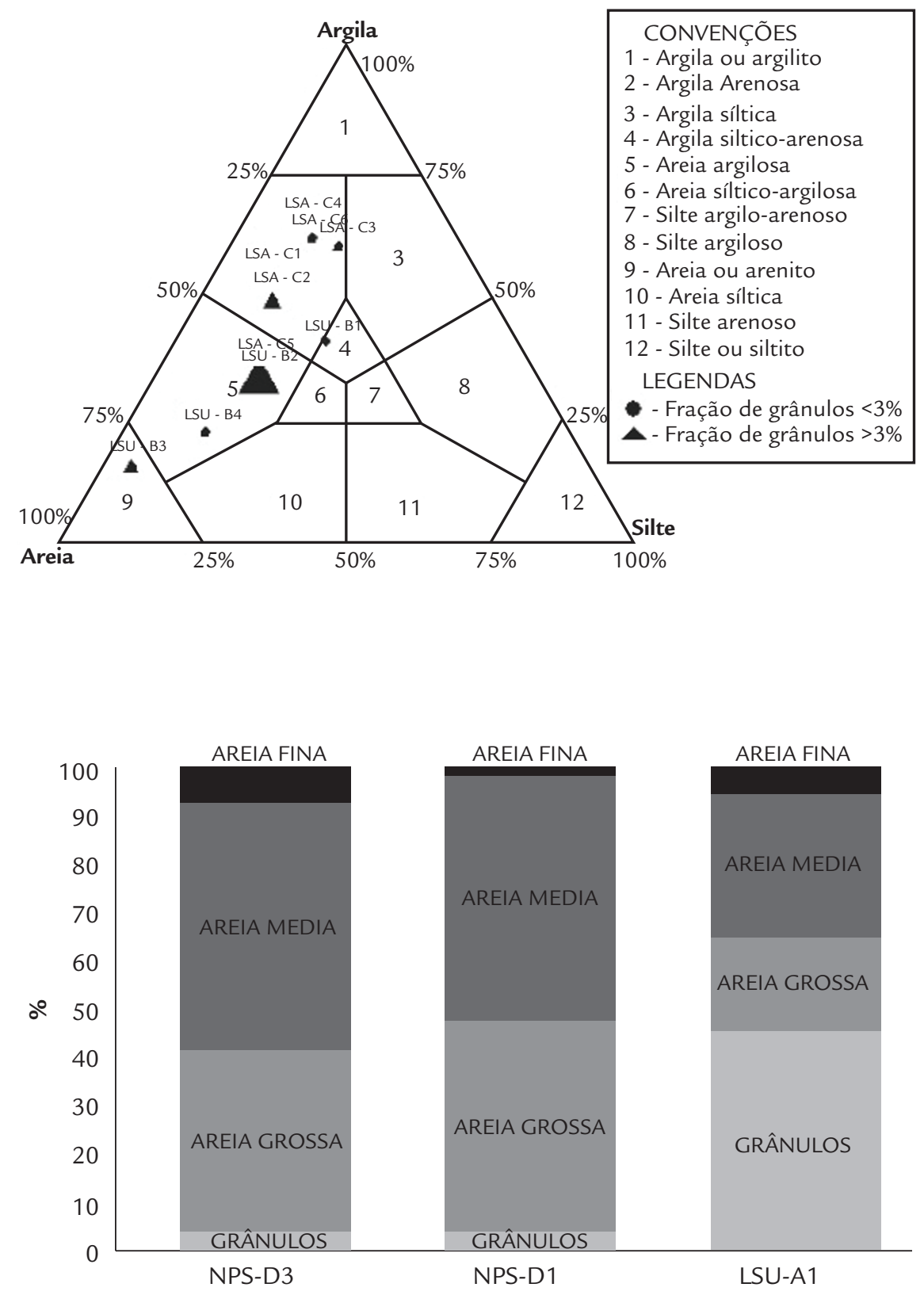


\subsection{Concentração de Matéria Orgânica}

É sabido que existe uma relação diretamente proporcional entre as concentrações de metais pesados e o teor da fração fina dos sedimentos, assim como com a concentração de matéria orgânica nos sedimentos (Paul et al. 1994, Cortecci 2005). Isso caracteriza propriedades geoquímicas importantes no processo da mobilização desses poluentes inorgânicos nos sedimentos. Dessa forma, os resultados das análises de matéria orgânica (Figura 4) obtidas na fração total dos sedimentos superficiais pertencentes às nascentes do rio Subaé podem ser usados para se fazer um prognóstico das concentrações possíveis de metais pesados nesse material.

Como pode se observar na Figura 3, as amostras que apresentaram altos conteúdos de matéria orgânica foram na nascente da Rua Pedro Suzart NPS-D1 (28\%), lagoa do Subaé LSU-B2 (25\%), e a lagoa Salgada LSA-C6 (20\%).

A lagoa Salgada foi o local que apresentou porcentagens de matéria orgânica homogênea (variou de $8-12 \%$ ) e relativamente mais enriquecida (excetuando-se dois pontos localizados, um, na lagoa do Subaé e, outro, na nascente da Rua Pedro Suzart). Esse ambiente foi dominado por partículas finas (silte e argila), o que está associado a uma maior área superficial do sedimento, permitindo um acúmulo de matéria orgânica. Percentagens mais altas de partículas finas no sedimento são comumente encontradas em sedimentos com altas concentrações de carbono orgânico, o que pode ser associado ao acúmulo de detritos advindos de fontes antrópicas (Porto Filho 1994). Para Petrúcio \& Furtado (1998), sedimentos predominantemente arenosos caracterizam-se por apresentarem reduzida concentração de carbono orgânico, ao contrário de partículas finas compostas por argila e silte, que apresentam maior teor de carbono orgânico e, consequentemente, maior será a tendência de adsorção de compostos orgânicos hidrofóbicos e de metais.

Já a nascente da Rua Pedro Suzart NPS-D1 que se distancia do ponto NPS-D3 em $500 \mathrm{~m}$, variou de $28 \%$ no D1 a $1 \%$ no NPS-D3. Como mencionado anteriormente, a estação de amostragem NPS-D1 recebe diretamente a descarga de efluentes domésticos, que, uma vez seguindo o curso do rio, pode sofrer degradação, decomposição e/ou sedimentação do material orgânico, ocasionando uma redução na concentração de MO no ponto NPS-D3 mais à jusante.

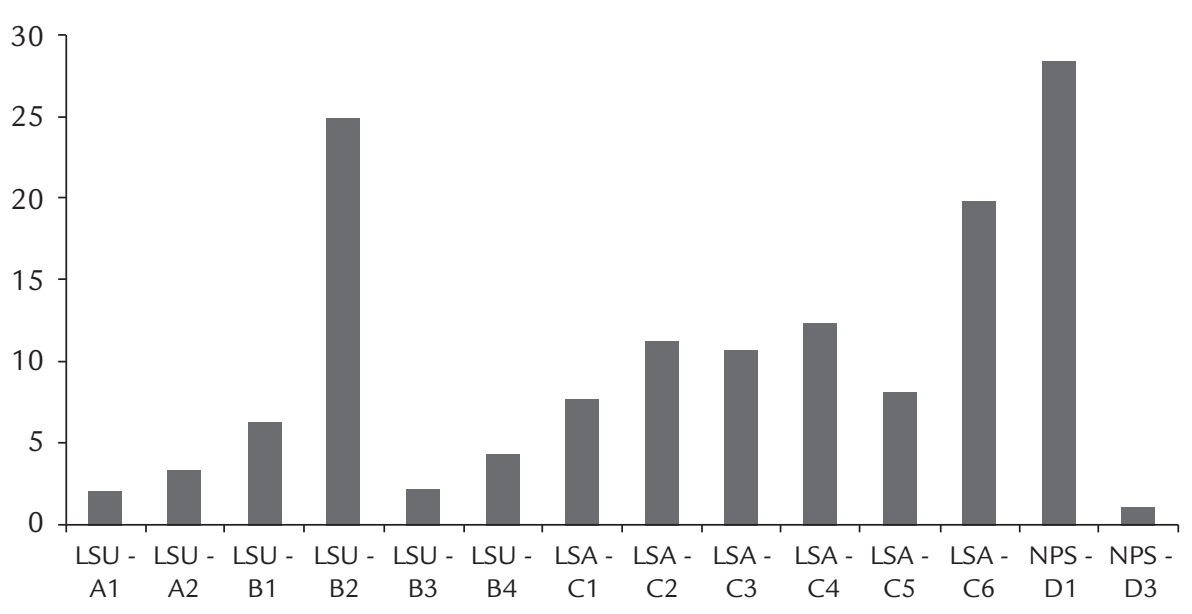

\subsection{Análise espectrorradiométrica infravermelho}

A região espectral do infravermelho de ondas curtas (1.200 a $3.000 \mathrm{~nm}$ ), ou SWIR (abreviatura do termo em inglês), é usada habitualmente para identificação mineral, visto que a maioria dos minerais apresenta assinatura espectral nessa região. Isso acontece porque, nessa região espectral, ocorrem os processos vibracionais (transições moleculares) que produzem absorções harmônicas fundamentais (Hunt 1977). Essas feições aparecem no espectro de reflectância como "depressões" e correspondem a regiões do intervalo de comprimento de ondas onde a luz é absorvida devido a ligações moleculares específicas do material alvo (Pontual et al. 1997). Os grupos moleculares que originam feições vibracionais na região do infravermelho de ondas curtas (SWIR) são: a água $\left(\mathrm{H}_{2} \mathrm{O}\right)$, a hidroxila $(\mathrm{OH})$, os car- bonatos e alguns fosfatos e sulfatos, sendo as feições da água e hidroxila ligada a um metal importantes na discriminação dos produtos de alteração hidrotermal e dos argilominerais (Meneses 1986). Nos espectros de minerais, rochas e solos, sempre que a água está presente, duas feições bem distintas aparecem: uma a $1.400 \mathrm{~nm}$ e outra a $1.900 \mathrm{~nm}$. A presença das bandas de absorção nessas posições indica a presença de moléculas de água dissociadas da estrutura mineral, como, por exemplo, a água de hidratação ou água capturada no retículo cristalino. O aparecimento da banda a $1.400 \mathrm{~nm}$ sem a banda a $1.900 \mathrm{~nm}$ informa, ainda, que se encontram presentes no material, grupos de $\mathrm{OH}$ diferentes da água como as hidroxilas. Essas são caracterizadas por feições de absorção próximas a $2.200 \mathrm{ou} 2.300 \mathrm{~nm}$ devido à presença 
de $\mathrm{Al}$ ou $\mathrm{Mg}$, respectivamente, associado ao íon $\mathrm{OH}-(\mathrm{Ma}-$ deira Netto \& Baptista 2000).

A caulinita $\left(\mathrm{Al}_{2} \mathrm{SiO}_{5}(\mathrm{OH})_{4}\right)$ é o argilomineral mais frequentemente encontrado nos solos tropicais, sendo formada pelo intemperismo das rochas ou como constituinte de rochas sedimentares. Suas principais feições estão associadas a vibrações moleculares das hidroxilas $(\mathrm{OH}-)$ em seu retículo cristalino. No infravermelho de ondas curtas, as feições mais perceptíveis estão localizadas em aproximadamente $2.200 \mathrm{~nm}$, que está relacionada à ligação $\mathrm{Al}-\mathrm{OH}$, e uma feição de absorção fraca próxima a $1.900 \mathrm{~nm}$, indicando a presença de água molecular absorvida. $\mathrm{Na}$ montmorilonita $\left((\mathrm{Al}, \mathrm{Mg})_{2-3}\right.$ $\left.\mathrm{Si}_{4} \mathrm{O}_{10}(\mathrm{OH})_{2} \cdot \mathrm{nH}_{2} \mathrm{O}\right)$, além da feição em $2.200 \mathrm{~nm}$ relacionada à ligação Al-OH, a feição em $1.900 \mathrm{~nm}$ está relacionada à água constitucional (Madeira Netto \& Baptista 2000).

Os espectros de reflectância na região do infravermelho obtidos em laboratório para as amostras coletadas na lagoa do Subaé, denominadas LSU-B1, LSU-B2, LSU-B3 e LSU-B4, apresentam feições de absorção na região do infravermelho de ondas curta em $\sim 2.208 \mathrm{~nm}$, indicativo da presença da molécula $\mathrm{Al}-\mathrm{OH} ; \mathrm{em} \sim 1.910 \mathrm{~nm}$, indicativo da presença de água $\left(\mathrm{H}_{2} \mathrm{O}\right)$ e em $\sim 1.410 \mathrm{~nm}$, indicando a presença da hidroxila $(\mathrm{OH})$. Essas feições de absorção são comumente encontradas nos argilominerais tais como caulinita e montmorilonita,

As feições de absorção da amostra LSU-B1 na região do infravermelho de ondas curtas em 2.208 e $2.165 \mathrm{~nm}$ (dupla), 1.911, 1.414 e $1.390 \mathrm{~nm}$ (dupla) e $2.385 \mathrm{~nm}$ estão representadas na Figura 5. Observa-se, na comparação entre espectro de reflectância da amostra e o espectro de reflectância da mistura de caulinita ( $62 \%)$ e montmorilonita ( 38\%) obtidos na biblioteca espectral do software de identificação mineral TSG ${ }^{\circledR}$, que as curvas apresentam similaridade espectral, indicando que a amostra é constituída por caulinita e montmorilonita nessas proporções. A amostra apresenta fator de reflectância máxima $<45 \%$.

A amostra LSU-B2 (Figura 6) apresenta feições de absorção principais em 2.208 e $2.175 \mathrm{~nm}$ (dupla), 1.916 e $1.413 \mathrm{~nm}$. O TSG ${ }^{\circledR}$ indicou apenas a presença de caulinita
Figura 5

Espectro de reflectância da amostra LSU-B1 comparado ao espectro de reflectância da biblioteca de referência da mistura dos argilominerais caulinita e montmorilonita.
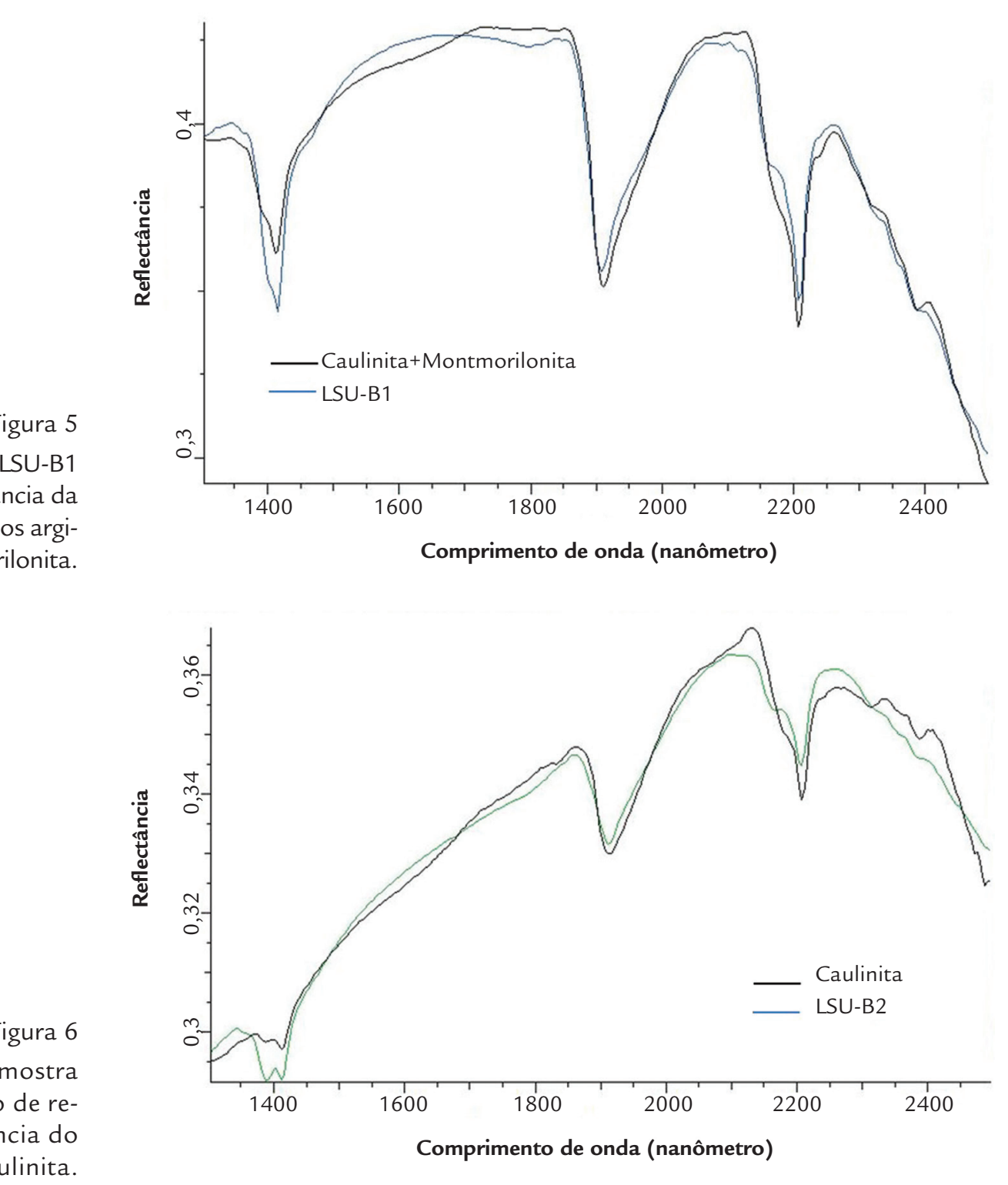

Espectro de reflectância da amostra LSU-B2 comparado ao espectro de reflectância da biblioteca de referência do argilomineral caulinita. 
na amostra com um erro médio de 469. Erros abaixo de 1000 são considerados normais, visto que as amostras das bibliotecas de referência são relativamente puras e as de campo, não. Outro fator relevante são os efeitos ópticos (como espalhamento) e as variações de cristalinidade da amostra (CSIRO 2012). A amostra apresenta fator de reflectância máxima $<40 \%$, possivelmente influenciado pela presença de matéria orgânica calculado em $24,89 \%$ (Figura 4). A presença da matéria orgânica influencia significativamente na reflectância, podendo exercer efeito de máscara, diminuindo as feições de absorção de outros constituintes do solo (Dalmolin 2005).

A amostra LSU-B3 apresenta bandas de absorção na região espectral do infravermelho de ondas curtas em 2.208 e $2.165 \mathrm{~nm}$ (dupla), $1.913 \mathrm{~nm}$ (simples), 1.414 e $1.390 \mathrm{~nm}$ (dupla), e $2.385 \mathrm{~nm}$ (Figura 7). Segundo o TSG ${ }^{\circledR}$, a amostra é constituída por $69 \%$ de caulinita e $31 \%$ de muscovita, com erro médio de 77 pontos. Observa-se, na comparação entre a assinatura espectral amostra e o espectro de reflectância da mistura dos minerais caulinita e muscovita, obtidos na biblioteca espectral do software de identificação mineral $\mathrm{TSG}^{\circledR}$, que as curvas apresentam similaridade espectral, indicando que a amostra é constituída pela mistura desses dois minerais, nessas proporções. $\mathrm{O}$ fator de reflectância máximo é inferior a $40 \%$.

A amostra LSU-B4 apresenta feições de absorção na região do infravermelho de ondas curtas em 2.209, 1.910, 1.415 e $2.296 \mathrm{~nm}$, conforme se observa na Figura 8. A presença de feição espectral próxima a $2.290 \mathrm{~nm}$ é devida à presença da molécula $\mathrm{Fe}-\mathrm{OH}$ na amostra, enquanto que a feição em $2.209 \mathrm{~nm}$ está relacionada à presença da molécula Al-OH encontrada em filossilicatos como a montmorilonita. $\mathrm{O}$ programa $\mathrm{TSG}^{\circledR}$ indicou a presença de montmorilonita $(60 \%)$ e siderita $(40 \%)$, um carbonato de ferro $\left(\mathrm{Fe}_{2} \mathrm{CO}_{3}\right)$.
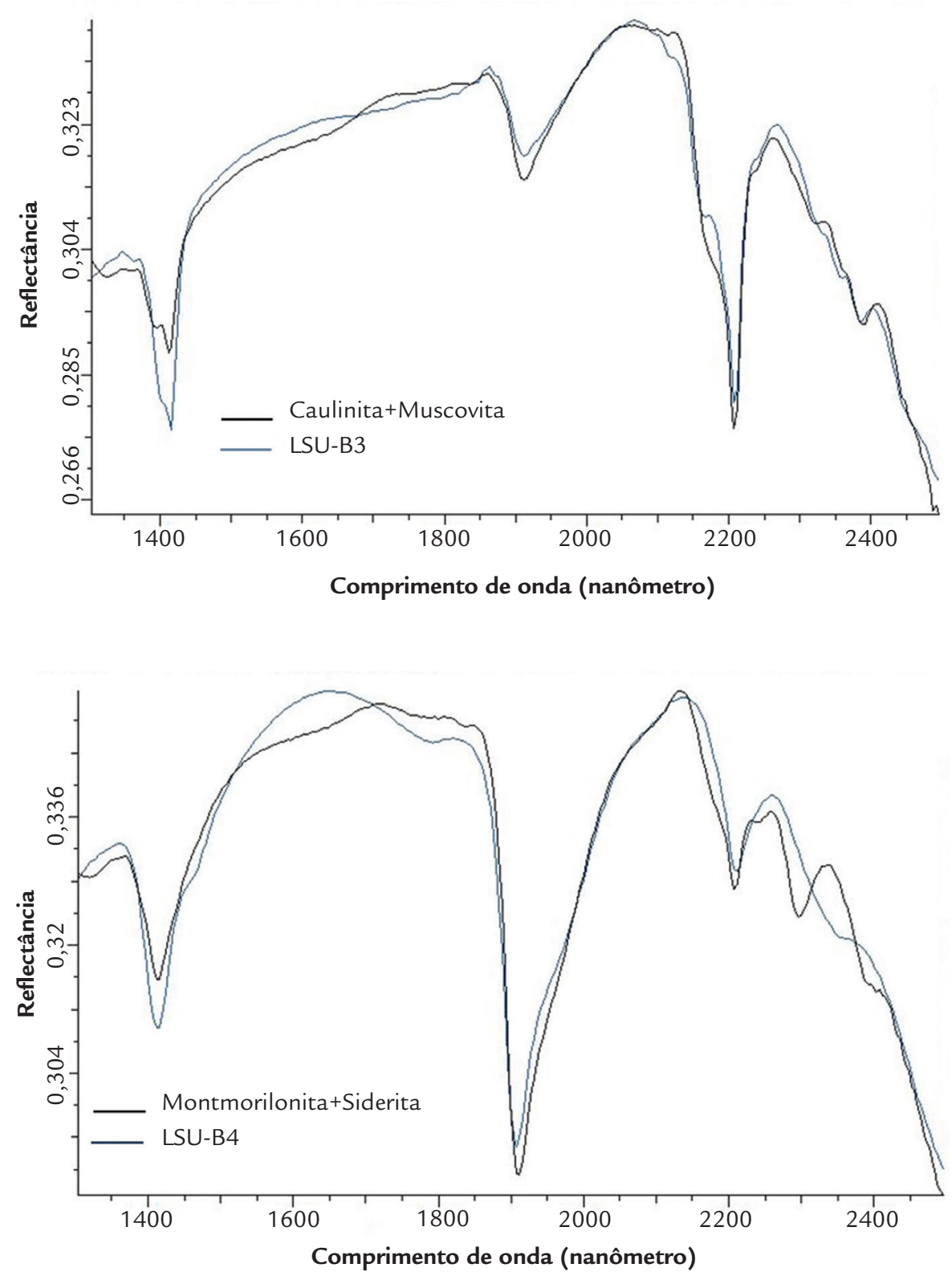

Figura 7

Espectro de reflectância da amostra LSU-B3 comparado ao espectro de reflectância da biblioteca de referência da mistura dos minerais caulinita e muscovita.
Figura 8

Espectro de reflectância da amostra LSU-B4 comparado ao espectro de reflectância da biblioteca de referência da mistura dos minerais montmorilonita e siderita. 
A feição da ligação $\mathrm{Fe}-\mathrm{OH}$ pode ser devida à presença desse carbonato rico em ferro ou indicar somente a presença do íon ferro na amostra. Apesar do erro médio calculado para a curva de referência ser de 87 pontos, um erro considerado muito baixo, é necessário uma investigação maior nessa amostra para confirmar a presença da siderita ou de outro mineral com a molécula $\mathrm{Fe}-\mathrm{OH}$ que estaria influenciando o espectro.

As amostras coletadas na Lagoa do Subaé às margens da BR-324, denominadas LSU-A1 e LSU-A2, apresentam feições de absorção em 2.208 e $2.172 \mathrm{~nm}$ (dupla), 1.910 $\mathrm{nm}$ (simples), em 1.413 e $1.321 \mathrm{~nm}$ (dupla) para a amostra LSU-A1, conforme ilustrado na Figura 9, e em 2.208 e $2.170 \mathrm{~nm}$ (dupla), $1.917,1.414$ e $2.387 \mathrm{~nm}$, para a amostra LSU-A2 (Figura 10).

A amostra LSU-A1 apresenta curva de reflectância espectral compatível com a presença de caulinita $(57 \%)$ e montmorilonita $(43 \%)$. Nessa amostra, o erro médio associado foi de 221 pontos e fator de reflectância inferior a $32 \%$. Para a amostra LSU-A2 o TSG ${ }^{\circledR}$ indicou apenas a presença de caulinita na amostra, com erro médio de 183 pontos e fator de reflectância inferior a $25 \%$. A amostra LSU-A1 apresenta $2 \%$ de matéria orgânica e a amostra LSU-A2, 3\% (Figura 4). Esses valores são coerentes com os valores de reflectância obtidos nas amostras, visto que, a amostra LSU-A2 apresentou menor fator de reflectância e maior percentual de $\mathrm{MO}$, indicando a relação direta entre a quantidade de matéria orgânica e a diminuição no fator de reflectância (Tabela 1).

Os espectros de reflectância obtidos em laboratório para as amostras coletadas na Lagoa Salgada, denominadas LSAC1, LSAC2, LSAC3, LSAC4, LSAC5 e LSAC6, estão representados na Figura 11. Todas as amostras apresentaram feições de absorção associadas à presença de caulinita e montmorilonita, nas amostras LSAC1, LSAC2, LSAC3 e LSAC4, em diferentes proporções. As amostras LSAC5 e LSAC6 apresentaram espectros de reflectância compatíveis somente com a presença de caulinita. $\mathrm{O}$ erro médio associado às misturas ficou abaixo de 125 para
Figura 9

Espectro de reflectância da amostra LSU-A1 comparado ao espectro de reflectância da biblioteca de referência da mistura dos minerais caulinita e montmorilonita.

Figura 10

Espectro de reflectância da amostra LSU-A2 comparado ao espectro de reflectância da biblioteca de referência da mistura do mineral caulinita.
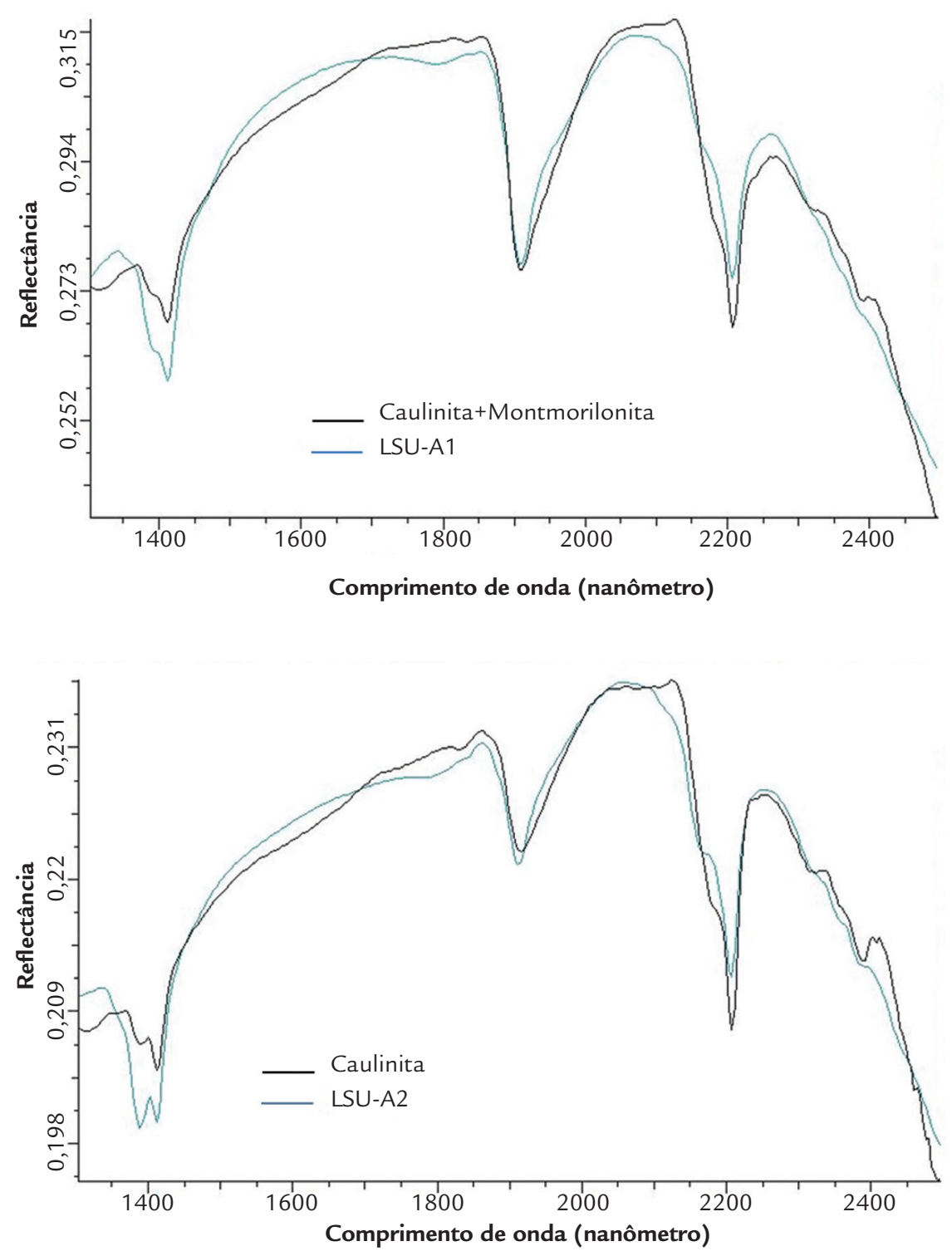
as quatro primeiras amostras, 217 para a amostra LSAC5 e 414 para a amostra LSAC6. Conforme informado anteriormente, erros abaixo de 1000 são considerados normais. A Tabela 2 apresenta as feições de absorção encontradas nas amostras da Lagoa Salgada (LSAC1 a LSAC6) bem como a mineralogia associada e a respectiva proporção de minerais na mistura.

As amostras NPS-D1 e NPS-D3, da nascente da Rua Pedro Suzart, apresentam feições de absorção associadas à presença de caulinita nas duas amostras, com feições de absorção diagnósticas em 2176 e 2208 nm (dupla) e feições adicionais em 1413, 1918 e 2485 nm. As amostras apresentam fator de reflectância menor que $25 \%$. O erro médio associado foi de 351 pontos para a amostra NPS-D1 e 255 pontos para a amostra NPS-D3. A Figura 12 apresenta os espectros de reflectância das amostras NPS-D1 e NPS-D3 respectivamente.

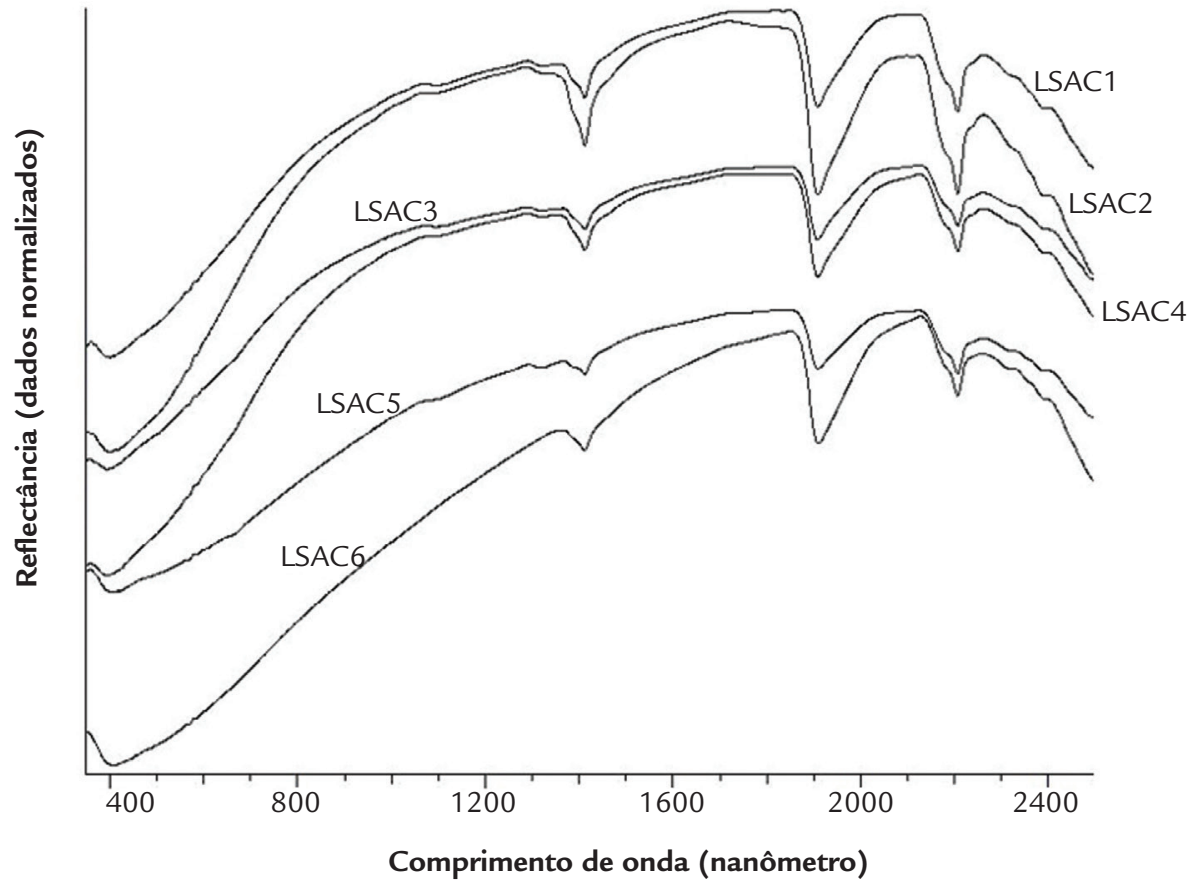

\begin{tabular}{|c|c|c|c|}
\hline Amostra & Feições de absorção (nanômetro) & Mineralogia associada & Proporção (\%) \\
\hline \multirow{2}{*}{ LSAC1 } & 1321 e 1413 (dupla), 1909, 2172 e & Caulinita & 51 \\
\hline & 2208,2385 (dupla) & Montmorilonita & 49 \\
\hline \multirow{2}{*}{ LSAC2 } & 1387 e 1413 (dupla), 1910, 2172 e & Caulinita & 53 \\
\hline & 2208 (dupla), 2385 & Montmorilonita & 47 \\
\hline \multirow{2}{*}{ LSAC3 } & 1321 e 1413 (dupla), 1910, 2175 e & Montmorilonita & 55 \\
\hline & 2208 (dupla), 2386 & Caulinita & 45 \\
\hline \multirow{2}{*}{ LSAC4 } & \multirow{2}{*}{$1413,1910,2176$ e 2208 (dupla) } & Montmorilonita & 55 \\
\hline & & Caulinita & 45 \\
\hline LSAC5 & $\begin{array}{c}1324 \text { e } 1413 \text { (dupla), 1910, } 2174 \text { e } \\
2208 \text { (dupla), } 2482\end{array}$ & Caulinita & 100 \\
\hline LSAC6 & $\begin{array}{c}1413,1912,2174 \text { e } 2208 \text { (dupla), } \\
2311\end{array}$ & Caulinita & 100 \\
\hline
\end{tabular}

Figura 11

Espectros de reflectância das amostras de sedimentos coletadas na Lagoa Salgada, Feira de Santana (BA).

\section{Tabela 1}

Feições de absorção das amostras de sedimentos superficiais, coletados na Lagoa Salgada, Feira de Santana (BA). 
Figura 12

Espectro de reflectância da amostra NPS-D1 e NPS-D2, respectivamente, nascente Subaé em Feira de Santana (BA), comparado ao espectro do mineral caulinita da biblioteca de referência.
Tabela 2

Sínteses dos resultados de mineralogia obtida por análise espectrorradiométrica e de matéria orgânica das amostras dos sedimentos superficiais das nascentes do rio Subaé- Feira de Santana (BA).
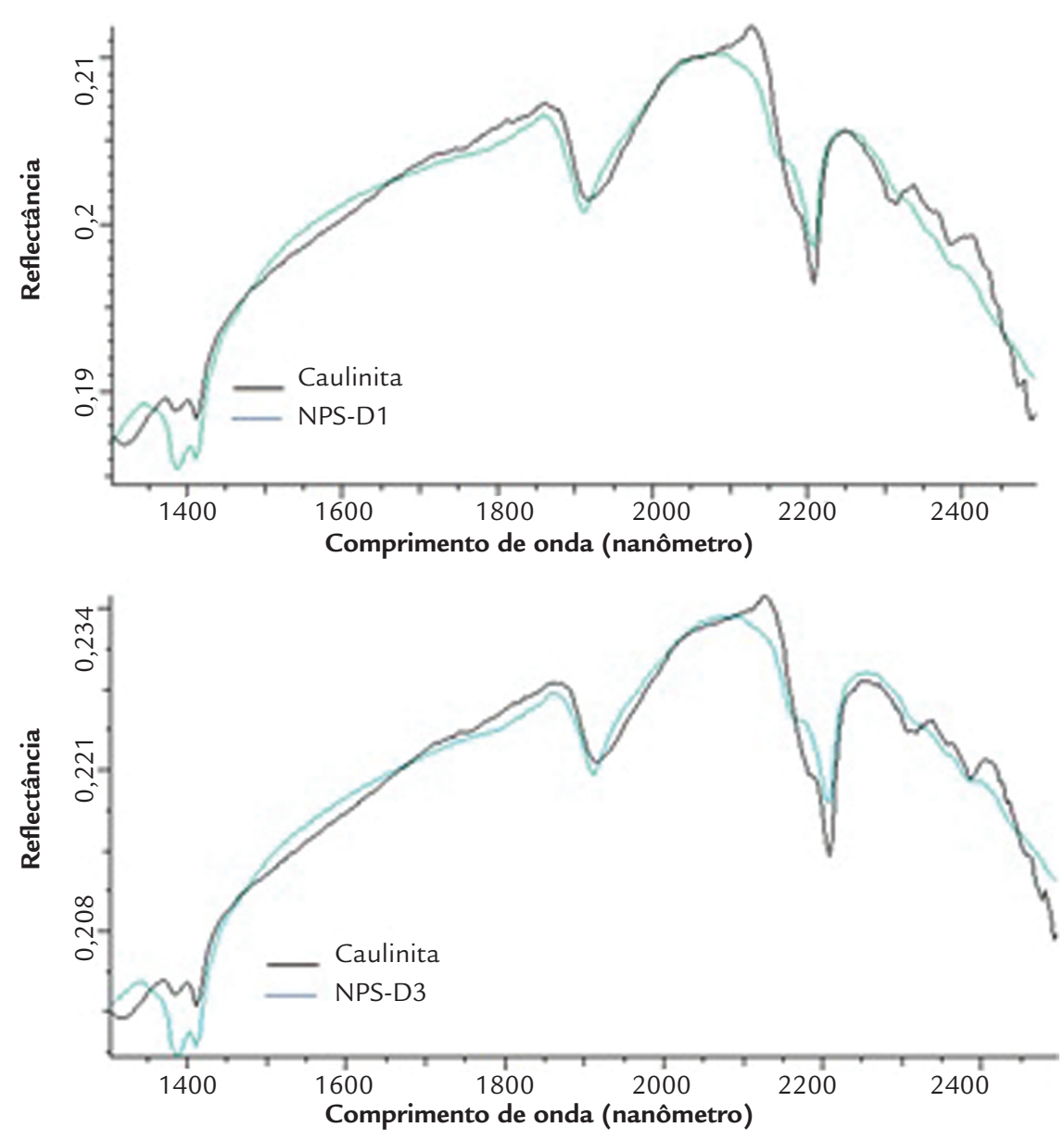

\begin{tabular}{|c|c|c|c|}
\hline Amostra & $\begin{array}{c}\text { Feições de absorção } \\
\text { (nanômetro) }\end{array}$ & Mineralogia associada & $\begin{array}{c}\text { Matéria Orgânica } \\
(\%)\end{array}$ \\
\hline LSU-A1 & $\begin{array}{c}2208 \text { e } 2172 \text { (dupla); 1910; } \\
1413 \text { e } 1321 \text { (dupla) }\end{array}$ & $\begin{array}{c}\text { Caulinita + Montmori- } \\
\text { lonita }\end{array}$ & 2 \\
\hline $\mathrm{LSU}-\mathrm{A} 2$ & $\begin{array}{c}2208 \text { e } 2170 \text { (dupla); } 1917 \\
1414 ; 2387\end{array}$ & Caulinita & 3 \\
\hline LSU-B1 & $\begin{array}{l}2208 \text { e } 2165 \text { (dupla); } 1911, \\
1414 \text { e } 1390 \text { (dupla); } 2385 .\end{array}$ & $\begin{array}{c}\text { Caulinita + Montmori- } \\
\text { lonita } \\
\end{array}$ & 6,3 \\
\hline LSU-B2 & $\begin{array}{c}2208 \text { e } 2175 \text { (dupla); } 1916 \\
1413\end{array}$ & Caulinita & 24,89 \\
\hline LSU-B3 & $\begin{array}{l}2208 \text { e } 2165 \text { (dupla); } 1913 \text {, } \\
1414 \text { e } 1390 \text { (dupla); } 2385 .\end{array}$ & Caulinita + Muscovita & 21,4 \\
\hline LSU-B4 & $2209 ; 1910 ; 1415 ; 2296$. & Montmorilonita + Siderita & N.O. \\
\hline LSAC1 & $\begin{array}{l}1321 \text { e } 1413 \text { (dupla), } 1909 \\
2172 \text { e } 2208,2385 \text { (dupla) }\end{array}$ & $\begin{array}{c}\text { Caulinita } \\
\text { Montmorilonita }\end{array}$ & $7,6-19,7$ \\
\hline LSAC2 & $\begin{array}{l}1387 \text { e } 1413 \text { (dupla), } 1910 \\
2172 \text { e } 2208 \text { (dupla), } 2385\end{array}$ & $\begin{array}{c}\text { Caulinita } \\
\text { Montmorilonita }\end{array}$ & $7,6-19,7$ \\
\hline LSAC3 & $\begin{array}{l}1321 \text { e } 1413 \text { (dupla), } 1910 \\
2175 \text { e } 2208 \text { (dupla), } 2386\end{array}$ & $\begin{array}{c}\text { Montmorilonita } \\
\text { Caulinita }\end{array}$ & $7,6-19,7$ \\
\hline LSAC4 & $\begin{array}{c}1413,1910,2176 \text { e } 2208 \\
\text { (dupla) }\end{array}$ & $\begin{array}{c}\text { Montmorilonita } \\
\text { Caulinita }\end{array}$ & $7,6-19,7$ \\
\hline LSAC5 & $\begin{array}{l}1324 \text { e } 1413 \text { (dupla), } 1910 \\
2174 \text { e } 2208 \text { (dupla), } 2482\end{array}$ & Caulinita & $7,6-19,7$ \\
\hline LSAC6 & $\begin{array}{c}\text { 1413, 1912, } 2174 \text { e } 2208 \\
\text { (dupla), } 2311\end{array}$ & Caulinita & $7,6-19,7$ \\
\hline NPS-D1 & $\begin{array}{c}2176 \text { e } 2208 \text { (dupla); } 1413 \\
1918 ; 2485\end{array}$ & Caulinita & N.O. \\
\hline NPS-D3 & $\begin{array}{c}2176 \text { e } 2208 \text { (dupla); } 1413 ; \\
1918 ; 2485\end{array}$ & Caulinita & N.O. \\
\hline
\end{tabular}

N.O.: Não Obtido 


\section{CONCLUSÕES}

Os minerais predominantes encontrados nas áreas de estudo, são a caulinita e esmectita, sendo que as amostras de sedimento que contém esses minerais apresentam diferenças nas quantidades de matéria orgânica $(7,6-19,7 \%)$ nas diversas porções da área investigada neste trabalho. A lagoa Salgada apresenta maior vulnerabilidade à contaminação por metais pesados pelo fato de apresentar sedimentos com maior quantidade de matéria orgânica e de material de granulometria fina. Além disso, os minerais (caulinita e esmectita, os mais comuns nos pontos analisados) que compõem as amostras de sedimento dessa lagoa possuem conhecidamente menor capacidade de adsorção, o que permite a dissolução de íons metálicos na coluna d'água, e, assim, por sua vez, o risco potencial de bioacumulação pela biota local. A técnica espectrorradiométrica em sedimentos lacustres é pouco utilizada por outros pesquisadores para as análises mineralógicas e os resultados obtidos por este trabalho, através dessa técnica, podem auxiliar na disseminação da mesma e possibilitar a divulgação das curvas adquiridas para fazer parte da biblioteca espectral e possivelmente ser utilizadas para comparações com outros sedimentos lacustres. Cabe salientar que o uso dessa técnica deveria ser acoplado com o de outras, como difratometria de raios-x, microscópica eletrônica de varredura com EDS, ou lupa binocular, para confirmar melhor as fases minerais indicadas pela espectrorradiometria.

\section{AGRADECIMENTOS}

À agência financiadora do estudo, FAPESB, através do Edital Pró-Pesquisa 011/2011, Nº 6937/2011. Ao Laboratório de Tecnologia da UEFS por ceder o espaço físico.

\section{REFERÊNCIAS}

Alloway B.J. \& Ayres, D.C. 1997. Chemical Principles of Environmental Pollution, Chapman and Hall: New York, 382 p.

ABNT - Associação Brasileira de Normas Técnicas. 1982. Solo: análise granulométrica - NBR-7181. Rio de Janeiro, 16p.

Barbosa J.S.F. \& Dominguez J.M.L. (Coords.). 1996. Geologia da Bahia: texto explicativo para o mapa geológico ao milionésimo. Salvador: superintendência de Geologia e Recursos Minerais, 400 p.

Brandão T.F. \& Santos R.L. 2010. Classificação climática do município de Feira de Santana, BA, através da análise frequencial e técnica dos quantis. In: Anais do XIV Seminário de Iniciação Científica da Universidade Estadual de Feira de Santana, UEFS, Feira de Santana.

Camargo G.M. 2006. SysGram: um sistema de código aberto para análises granulométricas do sedimento. Revista Brasileira de Geociencias, 36(2):371-378.

Clark R.N. 1999. Spectroscopy of Rocks and Minerals, and Principles of Spectroscopy. In: Rencz A.N. (Ed.). Manual of Remote Sensing, v. 3, Remote Sensing for the Earth Sciences, John Wiley and Sons, New York, p. 3-58.

Cortecci G. 2005. Geologia e Saúde. Tradução Wilson Scarpelli. PGAGEM. São Paulo. Disponível em: www.cprm.gov.br/pgagem/geosaude.pdf. Acessado em out 2011.

Dalmolin R.S.D., Gonçalves C.N., Klam E., Dick D.P. 2005. Relação entre os Constituintes do Solo e seu Comportamento Espectral. Revista Ciência Rural, 35(2):481-489.

Fisher S.G., Gray L.J., Grimm N.B., Busch D.E. 1982. Temporal succession in a desert stream ecosystem following flash flooding. Ecological Monographs, 52:93-110.

Folk R.L. \& Ward W.C. 1957. Brazos river bar: a study of significante of grain size parameters. Journal of Sedimentary Petrology, 27:3-26.

Franca-Rocha W.J.S. \& Nolasco M.C. 1998. Projeto Nascentes - Um olhar sobre Feira de Santana, Universidade Estadual de Feira de Santana, cd_Rom. 
Gatti L.V. 1997. Distribuição de Metais em Testemunhos de Sedimentos de Duas Lagoas Marginais do Rio Mogi-Guaçu (E.E de Jataí, Luiz Antônio, SP). Tese de Doutorado, São Carlos (SP). 130 p.

Gibbs R.J.1977. Transport phases of transition metals in the Amazon and Yukon Rivers. Geological Society of America Bulletin, 88:829-843.

Grove C.I., Paylor Ii E.D., Hook S.J. 1992. Laboratory Reflectance Spectra of 160 Minerals, 04. To 2.5 Micrometers. Jpl Publication 92-2, NASA - National Aeronautics And Space Administration, Jet Propulsion Laboratory. California, USA.

Hunt G.R. 1977. Spectral Signatures of Particulate Minerals in the Visible and Near Infrared. Geophysics, 42(3):501-513.

Hunt G.R. \& Ashley R.P. 1979. Spectra of Altered Rocks in the Visible and Near Infrared. Economic Geology, 74:1613-1629.

Jones B.F. \& Bowser C.J. 1978. The mineralogy and related chemistry of lake sediments. In: Lerman A. (Ed.). Lakes: Chemistry, Geology, and Physics. New York, Springer-Verlag, p. 179-235.

Lemes M.J.L., Figueiredo Filho P.M., Pires M.A.F. 2003. Influência da mineralogia dos sedimentos das bacias hidrográficas dos rios MogiGuaçu e Pardo na composição química das águas de abastecimento público. Quimica Nova, 26(1):13-20.

Maia L.P., Lacerda L.D., Monteiro L.H.U., Souza G.M. 2005. Estudo das Áreas de Manguezais do Litoral Nordeste do Brasil, Fortaleza: LABOMAR/ABCC/ISME-BR, 77 p.

Manahan S.E. 2000. Environmental Chemistry, $9^{\text {th }}$ ed. CRC Press LLC: USA, $753 \mathrm{p}$.

Paul A.C., Parameswaran M., Pillai K.C. 1994. Trace metal and lanthanides in a tropical river environment. Water Ar and Soil Pollution, 74:141-153.

Petrucio M.M. \& Furtado A.L.S. 1998. Concentração de Nitrogênio e Fósforo na Coluna D’água da Lagoa Imboassica. In: Ecologia das Lagoas Costeiras do Parque Nacional da Restinga de Jurubatiba e do Município de Macaé (RJ). Núcleo de Pesquisas Ecológicas do Município de Macaé (NUPEM) - Universidade Federal do Rio de Janeiro. 464 p.

Pontual S., Merry N.J., Gamson P. 1997. Spectral Analysis Guides for Mineral Exploration: G-MEX Manual Practical Aplications Handbook, AusSpec International, 1, p. 1-169.

Porto-Filho E. 1994. O Manguezal do rio Caveiras, Biguaçu,SC- Um Estudo de Caso. II - A Geomorfologia e o Manejo do Ecossistema Costeiro. Geosul, 17:62-72.

Salomons W. \& Förstner U. 1984. Metals in the Hidrocycle. Berlin Springer Verlag. 340 p.

Santos C.B. 2006. Caracterização do Impacto na Qualidade das Águas Subterrâneas, Causado pela Disposição dos Resíduos Sólidos Urbanos no Aterro Municipal da Cidade de Feira de Santana - Ba. Dissertação de Mestrado, Programa de Pós-Graduação em Geoquímica e Meio Ambiente da Universidade Federal da Bahia. 125 p.

Silva A.C., Torrado P.V., Abreu Junior J.S. 1999. Métodos de Quantificação da Matéria Orgânica do Solo. Revista da Universidade de Alfenas, $5: 21-26$.

Souza L.L.E. 2011. Análise Granulométrica, textural e mineralógica de sedimentos da Região Sul de Itaboraí-RJ. Monografia apresentada a Universidade Rural do Rio de Janeiro. 65 p. 
Shepard F.P. 1954. Nomenclature based on sand-silt-clay ratios. Journal Sedimentary Petrology, 24:151-158.

Soares H.M.V.M., Boaventura R.A.R., Machado A.A.S.C., da Silva J.C.G.E. 1999. Sediments as monitors of heavy metal contamination in the Ave River Basin (Portugal): multivariate analysis of data. Experimental Pollution, 105:311-323.

Suguio K. 1982. Rochas sedimentares: propriedades, gênese e importância econômica. 2 ed. São Paulo: Edgard Blucher, 500 p.

Truckenbrodt W., Kotschoubey B., Hieronymus B. 1995. Aluminization: an important process in the evolution of Amazonian bauxites. Tav. ICSOBA, n. 2 , p. 27-42.

Wetzel R.G. 1983. Limnology. 2 ed. Chicago: Sauders College Publishing. 